\title{
Equivalence between the semirelativistic limit of the Dirac-Maxwell equations and the Breit-Pauli model in the mean-field approximation
}

\author{
Y. Hinschberger, A. Dixit, G. Manfredi, and P.-A. Hervieux* \\ Institut de Physique et Chimie des Matériaux de Strasbourg, Centre National de la Recherche Scientifique \\ and Université de Strasbourg BP 43, F-67034 Strasbourg, France
}

(Received 22 September 2014; published 6 January 2015)

\begin{abstract}
We demonstrate the equivalence between (i) the semirelativistic limit (up to second order in the inverse of the speed of light) of the self-consistent Dirac-Maxwell equations and (ii) the Breit-Pauli equations in the mean-field (Hartree-like) approximation. We explain how the charge and current densities that act as sources in the Dirac-Maxwell equations are related to the microscopic two-electron interactions of the Breit-Pauli model (spin orbit, spin-other-orbit, and spin-spin). The key role played by the second-order corrections to the charge density is clarified.
\end{abstract}

DOI: 10.1103/PhysRevA.91.012101

\section{INTRODUCTION}

Relativistic effects can play an important role in the electron dynamics in heavy atoms and molecules [1,2], dense plasmas [3], and condensed-matter systems excited with intense and ultrafast laser pulses [4,5]. In order to model the charge and spin dynamics, the use of ab initio methods is restricted to systems composed of few interacting electrons. For larger systems (such as metallic clusters or nanoparticles), one needs to employ relativistic effective-field models based on the Maxwell and Dirac equations in order to describe the field-matter interactions.

A system of $N$ charged particles interacting though electromagnetic fields can be described classically at the second order in powers of $1 / c$ (where $c$ is the speed of light) by means of the Darwin Hamiltonian [6,7]. Its quantum version is generally referred to as the Dirac-Breit Hamiltonian, whose semirelativistic limit (also up to $c^{-2}$ ) is the so-called Breit-Pauli Hamiltonian. When other quantum electrodynamic effects are negligible, it has been shown that the use of this model yields theoretical predictions that are in good agreement with the experiments $[1,8]$.

The Breit-Pauli model can be profitably used to point out different types of two-particle interactions involving the charges and the spins of the particles. Such information may be valuable to uncover new mechanisms in the physics of ultrafast phenomena involving the charge and the spin of the interacting electrons. Unfortunately, the exact solution of the Breit-Pauli model for a system containing many electrons (dense plasmas, nanoparticles, etc.) is impossible analytically, and its numerical solution is beyond the capability of present-day computers. In order to overcome this limitation, a first approximation would resort to a mean-field approach of the problem, which is usually referred to as the Hartree approximation. Mean-field models incorporate the global effect of the $N$-particle interactions into an effective (mean) field that acts on a one-particle Hamiltonian. They are therefore much simpler to solve, both analytically and numerically. We will call this approach the "Hartree-Breit-Pauli" model.

As a complementary approach, we developed in the past [9] a mean-field model incorporating quantum, spin, and rela-

\footnotetext{
${ }^{*}$ Corresponding author.
}

PACS number(s): 03.65.Pm, 31.15.xr, 31.15.aj

tivistic effects. The model can be derived as a semirelativistic expansion of the self-consistent Dirac-Maxwell equations at second order in $1 / c$ and is based on two-component wave functions. It is therefore considerably simpler than fully relativistic models relying on the Dirac equation. Further, this model preserves the mathematical structure of the Schrödinger or KohnSham equations [10], which have been implemented in many numerical codes with great computational sophistication.

The main goal of the present study is to establish the equivalence between the semirelativistic Dirac-Maxwell model [9] and the mean-field Hartree-Breit-Pauli equations. Given the complexity of the latter equations, such equivalence is no trivial matter.

Particular emphasis will be put on the effect of the sources of the electromagnetic fields in the Maxwell equations (charge and current density), which are the quantities generally used for the modeling of time-dependent magneto-optics phenomena [11]. We will show that the second-order corrections to the charge density are mandatory to recover the various spin terms (spin orbit, spin-other-orbit, and spin-spin) that appear in the Hartree-Breit-Pauli equations.

In Sec. II, we derive the Dirac-Breit and Breit-Pauli models in the Hartree approximation. In Sec. III, we present the nonrelativistic limit of the Dirac-Maxwell equations and establish its equivalence with the Hartree-Breit-Pauli model. Finally, conclusions are provided in Sec. IV.

Throughout this work, we shall use the relation $\epsilon_{0} \mu_{0} c^{2}=1$.

\section{THE BREIT-PAULI MODEL AND ITS MEAN-FIELD VERSION (HARTREE-BREIT-PAULI)}

\section{A. Retardation effects on the electron-electron interactions}

The electromagnetic interaction energy between two moving electrons $i$ and $j$ of charge $q_{i}=q_{j}=q=-e(e>0)$ is written as $U=\frac{1}{2} \sum_{i=1}^{2} \sum_{j \neq i}\left(q_{i} \Phi_{j}-q_{i} \mathbf{v}_{i} \cdot \mathbf{A}_{j}\right)$, where $\mathbf{v}_{i}$ is the velocity of electron $i$ and $\Phi_{j}$ and $\mathbf{A}_{j}$ are the scalar and vector potentials created by the electron $j$. In the Coulomb gauge and the quasistatic approximation, the latter expression leads to the Darwin Lagrangian $\mathcal{L}^{D}$ :

$$
\mathcal{L}^{D}=-\frac{\bar{e}^{2}}{r_{i j}}+\bar{e}^{2}\left(\frac{\mathbf{v}_{i} \cdot \mathbf{v}_{j}}{2 c^{2} r_{i j}}+\frac{\left(\mathbf{v}_{i} \cdot \mathbf{r}_{i j}\right)\left(\mathbf{v}_{j} \cdot \mathbf{r}_{i j}\right)}{2 c^{2} r_{i j}^{3}}\right),
$$


where $\bar{e}^{2} \equiv \frac{q_{i} q_{j}}{4 \pi \epsilon_{0}}$ and $r_{i j} \equiv\left|\mathbf{r}_{i j}\right|=\left|\mathbf{r}_{i}-\mathbf{r}_{j}\right|$. The above expression illustrates the semirelativistic character-at second order in $1 / c-$ of the electromagnetic interaction at this level of approximation [6,7]. It is worthwhile to stress that the analytical expression of the two-particle interaction up to order $c^{-4}$ is much more complicated [12].

Within the framework of relativistic quantum mechanics [13], the Darwin Lagrangian can be used to build the Breit Hamiltonian $H_{i j}^{B}$ by replacing the classical velocity $\mathbf{v}$ in Eq. (1) with the operator $c \boldsymbol{\alpha}$, where $\boldsymbol{\alpha}$ are the Dirac matrices [14,15]. The second term in Eq. (1) leads to

$$
H_{i j}^{B}=-\bar{e}^{2}\left(\frac{\boldsymbol{\alpha}_{i} \cdot \boldsymbol{\alpha}_{j}}{2 r_{i j}}+\frac{\left(\boldsymbol{\alpha}_{i} \cdot \mathbf{r}_{i j}\right)\left(\boldsymbol{\alpha}_{j} \cdot \mathbf{r}_{i j}\right)}{2 r_{i j}^{3}}\right) \equiv G_{i j}+J_{i j},
$$

where $G_{i j} \equiv-\frac{\bar{e}^{2} \boldsymbol{\alpha}_{i} \cdot \boldsymbol{\alpha}_{j}}{2 r_{i j}}$ and $J_{i j} \equiv-\frac{\bar{e}^{2}\left(\boldsymbol{\alpha}_{i} \cdot \mathbf{r}_{i j}\right)\left(\boldsymbol{\alpha}_{j} \cdot \mathbf{r}_{i j}\right)}{2 r_{i j}^{3}}$ are the Gaunt and the gauge term, respectively.

This substitution enables one to incorporate the spin degrees of freedom in the electromagnetic interaction through the Dirac matrices. However, the wave function in the Dirac formalism is a bispinor describing both the electron and the positron, whereas a low-energy semirelativistic theory should consider only electrons and neglect positrons (negative energy states). Thus, the Breit Hamiltonian must be transformed and projected onto the electron basis, which is described by two-component Pauli spinors. This feat was first achieved by Breit in 1929 [15]. The derivation may be also found in modern textbooks [8,16-18].

The diagonalization of the Breit Hamiltonian leads, in the low-energy limit, to the so-called Breit-Pauli Hamiltonian, $H_{i j}^{\mathrm{BP}}$, which contains all the interactions between the electron spins:

$$
\begin{aligned}
H_{i j}^{\mathrm{BP}}= & \underbrace{-\frac{\pi \hbar^{2} \bar{e}^{2}}{m^{2} c^{2}} \delta\left(r_{i j}\right)-\frac{\bar{e}^{2}}{2 m^{2} c^{2}}\left(\frac{\mathbf{p}_{i} \cdot \mathbf{p}_{j}}{r_{i j}}+\frac{\mathbf{r}_{i j} \cdot\left(\mathbf{p}_{j} \cdot \mathbf{r}_{i j}\right) \mathbf{p}_{i}}{r_{i j}^{3}}\right)}_{(\mathrm{i})}) \\
& +\underbrace{\frac{\hbar \bar{e}^{2}}{4 m^{2} c^{2}}\left(\boldsymbol{\sigma}_{j}+2 \boldsymbol{\sigma}_{i}\right) \cdot\left(\frac{\mathbf{r}_{i j}}{r_{i j}^{3}} \wedge \mathbf{p}_{j}\right)-\frac{\hbar \bar{e}^{2}}{4 m^{2} c^{2}}\left(\boldsymbol{\sigma}_{i}+2 \boldsymbol{\sigma}_{j}\right) \cdot\left(\frac{\mathbf{r}_{i j}}{r_{i j}^{3}} \wedge \mathbf{p}_{i}\right)}_{(\mathrm{ii})} \\
& -\underbrace{\frac{\hbar^{2} \bar{e}^{2}}{4 m^{2} c^{2}}\left(-8 \pi \frac{\boldsymbol{\sigma}_{i} \cdot \boldsymbol{\sigma}_{j}}{3} \delta\left(r_{i j}\right)-\frac{\boldsymbol{\sigma}_{i} \cdot \boldsymbol{\sigma}_{j}}{r_{i j}^{3}}+3 \frac{\left(\boldsymbol{\sigma}_{i} \cdot \mathbf{r}_{i j}\right)\left(\boldsymbol{\sigma}_{j} \cdot \mathbf{r}_{i j}\right)}{r_{i j}^{5}}\right)}_{\text {(iii) }} .
\end{aligned}
$$

The above expression exhibits three groups of terms.

(i) The first group represents the "spin-free" interactions. In this group, the first term is a contact term, while the two other terms (called orbit-orbit interaction) are the quantum analogs of the corresponding terms in the Darwin Lagrangian (1).

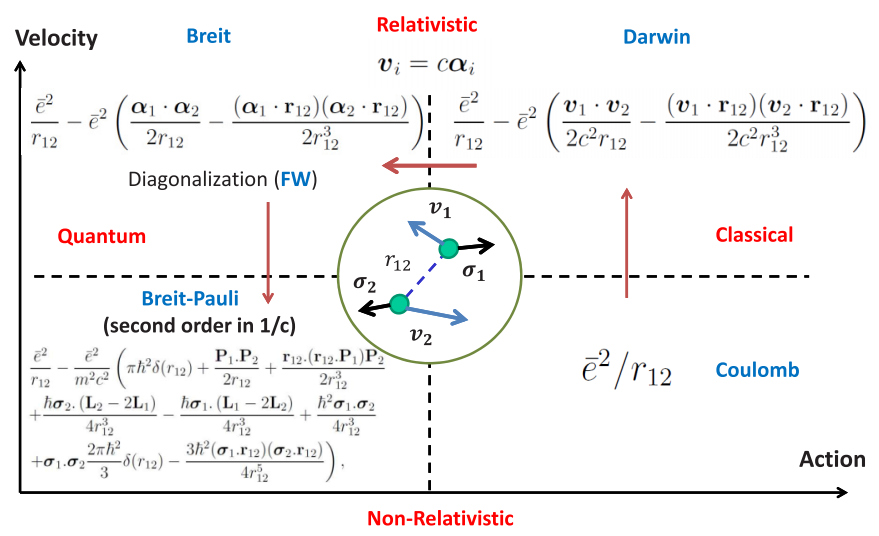

FIG. 1. (Color online) Steps of the procedure to obtain the BreitPauli Hamiltonian. Starting from the classical Darwin Hamiltonian (top left quarter) and moving counterclockwise one obtains the quantum Breit Hamiltonian (top left) and finally, at second order in $1 / c$, the Breit-Pauli Hamiltonian (bottom left). (ii) The second is the "spin-orbit" group, which contains the spin-orbit interaction $\boldsymbol{\sigma}_{i} \cdot\left(\mathbf{r}_{i j} \wedge \mathbf{p}_{i}\right)$ and $\boldsymbol{\sigma}_{j} \cdot\left(\mathbf{r}_{i j} \wedge \mathbf{p}_{j}\right)$, but also the so-called spin-other-orbit interaction $\boldsymbol{\sigma}_{i} \cdot\left(\mathbf{r}_{i j} \wedge \mathbf{p}_{j}\right)$ and $\sigma_{j} \cdot\left(\mathbf{r}_{i j} \wedge \mathbf{p}_{i}\right)$.

(iii) Finally, the third one is the "spin-spin" group, which displays the dipolar coupling between the two spin magnetic moments. Therefore, with the help of this procedure and within the framework of the relativistic quantum mechanics one is able to identify the microscopic mechanisms involving the charges and the spins of the two interacting electrons.

In summary, starting from the semirelativistic and classical Darwin Lagrangian, making use of the correspondence rule $\mathbf{v} \rightarrow c \boldsymbol{\alpha}$ and diagonalizing the Gaunt and gauge terms allowed us to build the electron-electron interaction Hamiltonian including all the relevant spin terms. We also stress that it is not possible to obtain this result simply by using the usual correspondence rule $m \mathbf{v}=\mathbf{p} \rightarrow-i \hbar \nabla$. The formal procedure is summarized schematically in Fig. 1.

\section{B. Hartree-Breit and Hartree-Breit-Pauli models}

In relativistic quantum mechanics, within the framework of the Dirac formalism, an electron is described with a bispinor $\Psi=(\phi, \chi)$, where $\phi$ and $\chi$ are, respectively, the electron and positron Pauli spinors. Considering the retardation effects 
introduced through the Breit Hamiltonian of Eq. (2), the Hamiltonian of an ensemble of $N$ interacting electrons may be written as

$$
\begin{aligned}
H & =\sum_{i=1}^{N} c \boldsymbol{\alpha}_{i} \cdot \mathbf{p}_{i}+m c^{2} \beta_{i}+\frac{1}{2} \sum_{i=1}^{N} \sum_{j \neq i}\left(\frac{\bar{e}^{2}}{r_{i j}}+H_{i j}^{B}\right) \\
& =\sum_{i=1}^{N} h_{i}+\frac{1}{2} \sum_{i=1}^{N} \sum_{j \neq i}\left(\frac{\bar{e}^{2}}{r_{i j}}+H_{i j}^{B}\right),
\end{aligned}
$$

where $h_{i}=c \boldsymbol{\alpha}_{i} \cdot \mathbf{p}_{i}+m c^{2} \beta_{i}$ is the single-electron Dirac Hamiltonian and $\boldsymbol{\alpha}_{i}$ and $\beta_{i}$ are the Dirac matrices [14]. In the Hartree approximation [19], by taking the total wave function as a product of $N$ bispinors $\Psi\left(\mathbf{r}_{1}, \ldots, \mathbf{r}_{N}\right)=$ $\Psi_{1}\left(\mathbf{r}_{1}\right) \Psi_{2}\left(\mathbf{r}_{2}\right) \ldots \Psi_{N}\left(\mathbf{r}_{N}\right)$ and applying Lagrange's method of undetermined multipliers, each bispinor $\Psi_{i}\left(\mathbf{r}_{i}\right) \equiv \Psi(\mathbf{x})$ is a solution of a single-particle Dirac equation:

$$
\left[c \boldsymbol{\alpha} \cdot \mathbf{p}+m c^{2} \beta+U_{\mathrm{eff}}^{B}(\mathbf{x})\right] \Psi(\mathbf{x})=i \hbar \frac{\partial}{\partial t} \Psi(\mathbf{x}),
$$

where $U_{\text {eff }}^{B}(\mathbf{x})$ is a mean-field potential created by all the other electrons. Writing $\mathbf{r}=\mathbf{r}_{i j} \equiv\left(\mathbf{x}-\mathbf{x}^{\prime}\right)$ and $r=\mid \mathbf{x}-$ $\mathbf{x}^{\prime} \mid, \Psi_{j}=\Psi_{j}\left(\mathbf{r}_{j}\right)$, the mean-field potential $U_{\text {eff }}^{B}(\mathbf{x})$ reads as

$$
\begin{aligned}
U_{\mathrm{eff}}^{B}(\mathbf{x})= & \bar{e}^{2} \sum_{j \neq i} \int d \mathbf{x}^{\prime} \frac{\Psi_{j}^{\dagger}\left(\mathbf{x}^{\prime}\right) \Psi_{j}\left(\mathbf{x}^{\prime}\right)}{\left|\mathbf{x}-\mathbf{x}^{\prime}\right|}-\bar{e}^{2} \boldsymbol{\alpha} \cdot \sum_{j \neq i} \int d \mathbf{x}^{\prime} \\
& \times\left(\frac{\Psi_{j}^{\dagger}\left(\mathbf{x}^{\prime}\right) \boldsymbol{\alpha}_{j} \Psi_{j}\left(\mathbf{x}^{\prime}\right)}{2\left|\mathbf{x}-\mathbf{x}^{\prime}\right|}+\frac{\mathbf{r}\left(\Psi_{j}^{\dagger}\left(\mathbf{x}^{\prime}\right) \boldsymbol{\alpha}_{j} \Psi_{j}\left(\mathbf{x}^{\prime}\right) \cdot \mathbf{r}\right)}{2\left|\mathbf{x}-\mathbf{x}^{\prime}\right|^{3}}\right) .
\end{aligned}
$$

The above expression is composed of the usual Hartree term (first term) supplemented by another term that originates from the Breit interaction. This approach is usually completed by adding the exchange interaction coming from the Pauli exclusion principle, which is described by the Dirac-FockBreit equations [18].

The same procedure may be successfully applied in the framework of the nonrelativistic limit of the Dirac equation, where the electron is described by a two-component spinor $\phi$ obeying the Pauli equation. In this case, the Hamiltonian for the $N$ interacting electrons reads as

$$
H=\sum_{i=1}^{N}\left(\frac{\mathbf{p}_{i}^{2}}{2 m}-\frac{\mathbf{p}_{i}^{4}}{8 m^{3} c^{2}}+m c^{2}\right)+\frac{1}{2} \sum_{i=1}^{N} \sum_{j \neq i}\left(\frac{\bar{e}^{2}}{r_{i j}}+H_{i j}^{\mathrm{BP}}\right),
$$

where $H_{i j}^{\mathrm{BP}}$ is the Breit-Pauli Hamiltonian representing the electromagnetic interaction up to the second order in $1 / c$, which is given by Eq. (3). By taking the wave function in the Hartree approximation $\phi\left(\mathbf{r}_{1}, \ldots, \mathbf{r}_{N}\right)=$ $\phi_{1}\left(\mathbf{r}_{1}\right) \phi_{2}\left(\mathbf{r}_{2}\right) \ldots \phi_{N}\left(\mathbf{r}_{N}\right)$, one obtains the Hartree-Breit-Pauli model, where the spinor $\phi_{i}\left(\mathbf{r}_{i}\right)=\phi(\mathbf{x})$ is a solution of the single-particle Pauli equation:

$$
\left(\frac{\mathbf{p}^{2}}{2 m}+m c^{2}+U_{\mathrm{eff}}^{\mathrm{BP}}(\mathbf{x})\right) \phi(\mathbf{x})=i \hbar \frac{\partial}{\partial t} \phi(\mathbf{x}),
$$

where $U_{\text {eff }}^{\mathrm{BP}}(\mathbf{x})$ is the mean-field potential calculated from the Breit-Pauli Hamiltonian. After lengthy but straightforward calculations, the analytical expression of $U_{\mathrm{eff}}^{\mathrm{BP}}(\mathbf{x})$ is as follows:

$$
\begin{aligned}
& U_{\mathrm{eff}}^{\mathrm{BP}}(\mathbf{x})=\underbrace{\bar{e}^{2} \sum_{j \neq i} \int d \mathbf{x}^{\prime} \frac{\phi_{j}^{\dagger}\left(\mathbf{x}^{\prime}\right) \phi_{j}\left(\mathbf{x}^{\prime}\right)}{\left|\mathbf{x}-\mathbf{x}^{\prime}\right|}}_{\text {Hartree }}-\frac{\pi \hbar^{2} \bar{e}^{2}}{m^{2} c^{2}} \underbrace{\sum_{j \neq i} \int d \mathbf{x}^{\prime} \phi_{j}^{\dagger}\left(\mathbf{x}^{\prime}\right) \delta\left(\mathbf{x}-\mathbf{x}^{\prime}\right) \phi_{j}\left(\mathbf{x}^{\prime}\right)}_{\text {Contact }} \\
& -\frac{\bar{e}^{2}}{2 m^{2} c^{2}} \underbrace{\left(\sum_{j \neq i} \int d \mathbf{x}^{\prime} \phi_{j}^{\dagger}\left(\mathbf{x}^{\prime}\right) \frac{\mathbf{p}_{j}}{\left|\mathbf{x}-\mathbf{x}^{\prime}\right|} \phi_{j}\left(\mathbf{x}^{\prime}\right)+\sum_{j \neq i} \int d \mathbf{x}^{\prime} \phi_{j}^{\dagger}\left(\mathbf{x}^{\prime}\right) \frac{\mathbf{r} \cdot\left(\mathbf{p}_{j} \cdot \mathbf{r}\right)}{\left|\mathbf{x}-\mathbf{x}^{\prime}\right|^{3}} \phi_{j}\left(\mathbf{x}^{\prime}\right)\right) \cdot \mathbf{p}}_{\text {Spin free }} \\
& -\frac{\hbar \bar{e}^{2}}{4 m^{2} c^{2}} \underbrace{\boldsymbol{\sigma} \cdot\left(\sum_{j \neq i} \int d \mathbf{x}^{\prime} \phi_{j}^{\dagger}\left(\mathbf{x}^{\prime}\right) \frac{\mathbf{x}-\mathbf{x}^{\prime}}{\left|\mathbf{x}-\mathbf{x}^{\prime}\right|^{3}} \phi_{j}\left(\mathbf{x}^{\prime}\right)\right) \wedge \mathbf{p}}_{\text {Spin orbit }}+\frac{\hbar \bar{e}^{2}}{4 m^{2} c^{2}} \underbrace{\sum_{j \neq i} \int d \mathbf{x}^{\prime} \phi_{j}^{\dagger}\left(\mathbf{x}^{\prime}\right) \boldsymbol{\sigma}_{j} \cdot\left(\frac{\mathbf{x}-\mathbf{x}^{\prime}}{\left|\mathbf{x}-\mathbf{x}^{\prime}\right|^{3}} \wedge \mathbf{p}_{j}\right) \phi_{j}\left(\mathbf{x}^{\prime}\right)}_{\text {Spin orbit }} \\
& +\frac{2 \hbar \bar{e}^{2}}{4 m^{2} c^{2}} \underbrace{\sigma \cdot \sum_{j \neq i} \int d \mathbf{x}^{\prime} \phi_{j}^{\dagger}\left(\mathbf{x}^{\prime}\right)\left(\frac{\mathbf{x}-\mathbf{x}^{\prime}}{\left|\mathbf{x}-\mathbf{x}^{\prime}\right|^{3}} \wedge \mathbf{p}_{j}\right) \phi_{j}\left(\mathbf{x}^{\prime}\right)}_{\text {Spin-other-orbit }}+\frac{2 \hbar \bar{e}^{2}}{4 m^{2} c^{2}} \underbrace{\mathbf{p} \cdot \underbrace{}_{j \neq i} \int d \mathbf{x}^{\prime} \phi_{j}^{\dagger}\left(\mathbf{x}^{\prime}\right)\left(\sigma_{j} \wedge \frac{\mathbf{x}-\mathbf{x}^{\prime}}{\left|\mathbf{x}-\mathbf{x}^{\prime}\right|^{3}}\right) \phi_{j}\left(\mathbf{x}^{\prime}\right)}_{\text {Spin-other-orbit }} \\
& -\frac{\hbar^{2} \bar{e}^{2}}{4 m^{2} c^{2}} \underbrace{\sigma \cdot \sum_{j \neq i} \int d \mathbf{x}^{\prime} \phi_{j}^{\dagger}\left(\mathbf{x}^{\prime}\right)\left(-8 \pi \frac{\boldsymbol{\sigma}_{j}}{3} \delta\left(\mathbf{x}-\mathbf{x}^{\prime}\right)-\frac{\sigma_{j}}{\left|\mathbf{x}-\mathbf{x}^{\prime}\right|^{3}}+3 \frac{\mathbf{r}\left(\boldsymbol{\sigma}_{j} \cdot \mathbf{r}\right)}{\left|\mathbf{x}-\mathbf{x}^{\prime}\right|^{5}}\right) \phi_{j}\left(\mathbf{x}^{\prime}\right)}_{\text {Spin-spin }} .
\end{aligned}
$$

The origin of the various terms of the above expression is specified under the horizontal braces, following the notation used for Eq. (3). Let us note that the term $-p^{4} / 8 m^{3} c^{2}$ in Eq. (5) has been neglected (see explanation in the next section). 


\section{SEMIRELATIVISTIC LIMIT OF THE DIRAC-MAXWELL MODEL}

\section{A. Dirac-Maxwell model in the Coulomb gauge and quasistatic approximation}

In a quantum relativistic mean-field approach, the electron dynamics is governed by the Dirac equation, coupled selfconsistently to the Maxwell equations written in terms of the scalar and vector potentials $(\Phi, \mathbf{A})[20]$ in the Lorentz gauge $\left(\boldsymbol{\nabla} \cdot \mathbf{A}+\frac{1}{c} \frac{\partial \Phi}{\partial t}=0\right)$, and reads

$$
\begin{gathered}
{\left[c \boldsymbol{\alpha} \cdot(\mathbf{p}-q \mathbf{A})+m c^{2} \beta+q \Phi\right] \Psi=i \hbar \frac{\partial \Psi}{\partial t}} \\
-\Delta \Phi+\frac{1}{c^{2}} \frac{\partial^{2} \Phi}{\partial t^{2}}=\frac{q \rho}{\epsilon_{0}} \\
-\Delta \mathbf{A}+\frac{1}{c^{2}} \frac{\partial^{2} \mathbf{A}}{\partial t^{2}}=q \mu_{0} \mathbf{j},
\end{gathered}
$$

where the sources are expressed from the four-component Dirac current density as

$$
(\rho c, \mathbf{j})=c \sum_{i=1}^{N}\left(\Psi_{i}^{\dagger} \Psi_{i}, \Psi_{i}^{\dagger} \boldsymbol{\alpha} \Psi_{i}\right) .
$$

Equations (7)-(10) constitute a fully relativistic Lorentz covariant model for describing the quantum dynamics of a system of $N$ interacting electrons in the mean-field approximation.

In the present work, we prefer the use of the Coulomb gauge $(\boldsymbol{\nabla} \cdot \mathbf{A}=0)$ along with the quasistatic approximation $\left(\Delta \mathbf{A} \gg \frac{1}{c^{2}} \frac{\partial^{2} \mathbf{A}}{\partial t^{2}}\right)$. In this framework, the Maxwell equations (8) and (9) can be written in terms of two Poisson-like equations $[21,22]$ :

$$
\begin{gathered}
-\Delta \Phi=\frac{q \rho}{\epsilon_{0}}, \\
-\Delta \mathbf{A}=q \mu_{0} \mathbf{j}_{T},
\end{gathered}
$$

where $\mathbf{j}_{T}$ is the transverse component of the current density $\mathbf{j}$. Indeed, the current density can be decomposed into its transverse and longitudinal components $\mathbf{j}=\mathbf{j}_{L}+\mathbf{j}_{T}$ (by definition $\nabla \cdot \mathbf{j}_{T}=0$ and $\nabla \wedge \mathbf{j}_{L}=0$ ), which are defined as [7]

$$
\begin{gathered}
\mathbf{j}_{T}(\mathbf{x})=\frac{1}{4 \pi} \nabla \wedge \nabla \wedge \int \frac{d \mathbf{x}^{\prime} \mathbf{j}\left(\mathbf{x}^{\prime}\right)}{\left|\mathbf{x}-\mathbf{x}^{\prime}\right|}, \\
\mathbf{j}_{L}(\mathbf{x})=-\frac{1}{4 \pi} \nabla\left(\int \frac{d \mathbf{x}^{\prime} \nabla^{\prime} \cdot \mathbf{j}\left(\mathbf{x}^{\prime}\right)}{\left|\mathbf{x}-\mathbf{x}^{\prime}\right|}\right) .
\end{gathered}
$$

The analytical solutions of Eqs. (11) and (12) can be expressed as [21]

$$
\begin{gathered}
\Phi(\mathbf{x})=\frac{q}{4 \pi \epsilon_{0}} \int \frac{d \mathbf{x}^{\prime} \rho\left(\mathbf{x}^{\prime}\right)}{\left|\mathbf{x}-\mathbf{x}^{\prime}\right|} \\
\mathbf{A}(\mathbf{x})=\frac{q \mu_{0}}{4 \pi} \int \frac{d \mathbf{x}^{\prime}}{\left|\mathbf{x}-\mathbf{x}^{\prime}\right|}\left(\frac{1}{4 \pi} \nabla^{\prime} \wedge \nabla^{\prime} \wedge \int \frac{d \mathbf{x}^{\prime \prime} \mathbf{j}\left(\mathbf{x}^{\prime \prime}\right)}{\left|\mathbf{x}^{\prime}-\mathbf{x}^{\prime \prime}\right|}\right) \\
=\frac{q \mu_{0}}{4 \pi} \int d \mathbf{x}^{\prime}\left(\frac{\mathbf{j}\left(\mathbf{x}^{\prime}\right)}{2\left|\mathbf{x}-\mathbf{x}^{\prime}\right|}+\frac{\mathbf{r}\left[\mathbf{r} \cdot \mathbf{j}\left(\mathbf{x}^{\prime}\right)\right]}{2\left|\mathbf{x}-\mathbf{x}^{\prime}\right|^{3}}\right)
\end{gathered}
$$

where $\mathbf{r} \equiv \mathbf{x}-\mathbf{x}^{\prime}$. The vector potential $\mathbf{A}(\mathbf{x})$ appearing in Eq. (16) is called the Darwin vector potential. Indeed, if one considers a classical two-electron system, the electromagnetic energy between two particles of charges $q_{i}$ and $q_{j}$ reads $U=$ $q_{i} \Phi_{j}-q_{i} \mathbf{v}_{i} \cdot \mathbf{A}_{j}$. Writing the charge and current densities for a single classical particle as $\rho\left(\mathbf{x}^{\prime}\right)=\delta\left(\mathbf{x}^{\prime}\right)$ and $\mathbf{j}\left(\mathbf{x}^{\prime}\right)=\delta\left(\mathbf{x}^{\prime}\right) \mathbf{v}$ and plugging these expressions into Eqs. (15) and (16), the electromagnetic energy reads $U=\frac{q_{i} q_{j}}{4 \pi \epsilon_{0} r}-\frac{q_{i} q_{j}}{\left(4 \pi \epsilon_{0}\right) c^{2}}\left(\frac{\mathbf{v}_{i} \cdot \mathbf{v}_{j}}{2 r}+\frac{\left(\mathbf{v}_{i} \cdot \mathbf{r}\right)\left(\mathbf{r} \cdot \mathbf{v}_{j}\right)}{2 r^{3}}\right)$, which is precisely the Darwin Lagrangian of Eq. (1).

Hence, within the framework of the Coulomb gauge and the quasistatic approximation, the set of Eqs. (7)-(9) becomes

$$
\begin{gathered}
\left(c \boldsymbol{\alpha} \cdot \mathbf{p}+m c^{2} \beta+U_{\mathrm{eff}}^{s c}\right) \Psi=i \hbar \frac{\partial \Psi}{\partial t}, \\
\Delta \Phi=-\frac{q \rho}{\epsilon_{0}}, \\
\Delta \mathbf{A}=-\mu_{0} q \mathbf{j}_{T},
\end{gathered}
$$

where $U_{\text {eff }}^{s c}$ is the electromagnetic mean-field potential given by

$$
U_{\mathrm{eff}}^{s c}=q \Phi-q c \boldsymbol{\alpha} \cdot \mathbf{A} .
$$

By plugging the Dirac particle density $\rho\left(\mathbf{x}^{\prime}\right)=$ $\sum_{i=1}^{N} \Psi_{i}^{\dagger}\left(\mathbf{x}^{\prime}\right) \Psi_{i}\left(\mathbf{x}^{\prime}\right)$ and the Dirac current density $\mathbf{j}\left(\mathbf{x}^{\prime}\right)=\sum_{i=1}^{N} c \Psi_{i}^{\dagger}\left(\mathbf{x}^{\prime}\right) \boldsymbol{\alpha} \Psi_{i}\left(\mathbf{x}^{\prime}\right)$, respectively, into Eqs. (15) and (16), $U_{\text {eff }}^{s c}$ takes the form

$$
\begin{aligned}
U_{\mathrm{eff}}^{s c}(\mathbf{x})= & \bar{e}^{2} \sum_{i=1}^{N} \int d \mathbf{x}^{\prime} \frac{\Psi_{i}^{\dagger}\left(\mathbf{x}^{\prime}\right) \Psi_{i}\left(\mathbf{x}^{\prime}\right)}{\left|\mathbf{x}-\mathbf{x}^{\prime}\right|}-\bar{e}^{2} \boldsymbol{\alpha} \cdot \sum_{i=1}^{N} \int d \mathbf{x}^{\prime} \\
& \times\left(\frac{\Psi_{i}^{\dagger}\left(\mathbf{x}^{\prime}\right) \boldsymbol{\alpha}_{i} \Psi_{i}\left(\mathbf{x}^{\prime}\right)}{2\left|\mathbf{x}-\mathbf{x}^{\prime}\right|}+\frac{\mathbf{r}\left(\Psi_{i}^{\dagger}\left(\mathbf{x}^{\prime}\right) \boldsymbol{\alpha}_{i} \Psi_{i}\left(\mathbf{x}^{\prime}\right) \cdot \mathbf{r}\right)}{2\left|\mathbf{x}-\mathbf{x}^{\prime}\right|^{3}}\right) .
\end{aligned}
$$

The above expression is identical to the one of $U_{\text {eff }}^{B}$ for the Hartree-Breit model [Eq. (4)], with the exception of the selfinteraction term $(i=j)$. The self-consistent Dirac-Maxwell approach presented here is therefore completely equivalent to the usual method (based on Lagrange's multipliers and a variational principle) that we discussed in Sec. II B [19].

In the next subsection, we will extend this result to the semirelativistic version of the Dirac-Maxwell system and prove that it is equivalent to the semirelativistic version of the Hartree-Breit model, i.e., the Hartree-Breit-Pauli equations.

\section{B. Semirelativistic expansion of the Dirac-Maxwell model}

In this section we investigate the semirelativistic limit of the Dirac-Maxwell model, valid up to the second order in powers of $1 / c$. This low-energy semirelativistic theory considers only electrons and neglects positrons (negative energy states) and the Dirac bispinor is transformed in a two-component Pauli spinor $\Psi=(\phi, \chi) \rightarrow \phi$. Using the Foldy-Wouthuysen transformation $[9,23,24]$, the Dirac Hamiltonian in the presence of an electromagnetic field [Eq. (7)] can be expanded at second order in $1 / c$, to obtain

$$
\begin{aligned}
H= & m c^{2}+\frac{(\mathbf{p}-q \mathbf{A})^{2}}{2 m}+q \Phi-\frac{q \hbar}{2 m} \boldsymbol{\sigma} \cdot \mathbf{B}-\frac{q \hbar^{2}}{8 m^{2} c^{2}} \nabla \cdot \mathbf{E} \\
& -\frac{q \hbar}{8 m^{2} c^{2}} \boldsymbol{\sigma} \cdot(\mathbf{E} \wedge(\mathbf{p}-q \mathbf{A})-(\mathbf{p}-q \mathbf{A}) \wedge \mathbf{E}),
\end{aligned}
$$

where the electromagnetic fields are defined as usual as $\mathbf{E}=-\nabla \Phi-\frac{\partial \mathbf{A}}{\partial t}$ and $\mathbf{B}=\nabla \wedge \mathbf{A}$. Here, the first term on the 
right-hand side is the electron rest mass energy; the next two terms constitute the standard Schrödinger Hamiltonian in the presence of an electromagnetic field; the fourth term is the Pauli spin term (Zeeman effect); the $\nabla \cdot \mathbf{E}$ term is the Darwin term; and the last two terms represent the spin-orbit coupling.

We neglected the term $-\frac{(\mathbf{p}-q \mathbf{A})^{4}}{8 m^{3} c^{2}}$ in Eq. (22), which is the first relativistic correction to the electron mass (expansion of the Lorentz factor to second order in powers of $1 / c$ ). This assumption is motivated by the fact that the latter introduces fourth-order derivatives in the evolution equation [9], unlike the nonrelativistic Schrödinger equation, which contains only second-order derivatives.

Hence, in the nonrelativistic limit, the Dirac equation (7) is transformed into a Schrödinger equation for the twocomponent spinor $\phi$ with the Hamiltonian of Eq. (22). Further, it was shown in [9] that the sources $\rho$ and $\mathbf{j}$ in Eqs. (8) and (9) must also be expanded in powers of $1 / c$, such as $\rho=\rho^{(0)}+\rho^{(2)}$ and $\mathbf{j}=\mathbf{j}^{(0)}+\mathbf{j}^{(2)}$, with

$$
\begin{gathered}
\rho^{(0)}=\phi^{\dagger} \phi \\
\mathbf{j}^{(0)}=\frac{i \hbar}{2 m}\left(\phi \nabla \phi^{\dagger}-\phi^{\dagger} \nabla \phi\right)-\frac{q}{m} \phi^{\dagger} \phi \mathbf{A}+\frac{\hbar}{2 m} \nabla \wedge\left(\phi^{\dagger} \boldsymbol{\sigma} \phi\right) \\
\rho^{(2)}=\frac{\hbar^{2}}{8 m^{2} c^{2}} \nabla \cdot \nabla\left(\phi^{\dagger} \phi\right)-\frac{q \hbar}{4 m^{2} c^{2}} \nabla \cdot\left[\left(\phi^{\dagger} \boldsymbol{\sigma} \phi\right) \wedge \mathbf{A}\right] \\
-\frac{i \hbar^{2}}{8 m^{2} c^{2}} \nabla \cdot\left[\phi^{\dagger} \boldsymbol{\sigma} \wedge(\nabla \phi)+\left(\nabla \phi^{\dagger}\right) \wedge \boldsymbol{\sigma} \phi\right] \\
\mathbf{j}^{(2)}=-\frac{q \hbar}{4 m^{2} c^{2}}\left(\phi^{\dagger} \boldsymbol{\sigma} \phi\right) \wedge \mathbf{E}-\frac{\hbar^{2}}{8 m^{2} c^{2}} \partial_{t} \nabla\left(\phi^{\dagger} \phi\right) \\
+\frac{i \hbar^{2}}{8 m^{2} c^{2}} \partial_{t}\left[\phi^{\dagger} \boldsymbol{\sigma} \wedge(\nabla \phi)+\left(\nabla \phi^{\dagger}\right) \wedge \boldsymbol{\sigma} \phi\right] \\
+\frac{q \hbar}{4 m^{2} c^{2}} \partial t\left(\phi^{\dagger} \boldsymbol{\sigma} \phi \wedge \mathbf{A}\right) .
\end{gathered}
$$

It would be desirable to build a model that treats on the same footing (i.e., at the same order in powers of $1 / c$ ) both the equation of motion (Pauli) and the field equations (Maxwell). Thus, the Maxwell equations (8) and (9) should also be expanded at the second order in powers of $1 / c$ by writing the electromagnetic potentials in powers of $1 / c$ as $\Phi=\Phi^{(0)}+\Phi^{(2)}+\ldots$ and $\mathbf{A}=\mathbf{A}^{(0)}+\mathbf{A}^{(2)}+\ldots$ [25]. Since we work in the framework of the Coulomb gauge and the quasistatic approximation, the appropriate Maxwell equations are Eqs. (11) and (12). They can be expanded at second order of $1 / c$ as follows:

$$
\begin{aligned}
\mathbf{A}^{(0)} & =0, \\
-\Delta \Phi^{(0)} & =\frac{q \rho^{(0)}}{\epsilon_{0}}, \\
-\Delta \mathbf{A}^{(2)} & =\frac{q \mathbf{j}_{T}^{(0)}}{\epsilon_{0} c^{2}}, \\
-\Delta \Phi^{(2)} & =\frac{q \rho^{(2)}}{\epsilon_{0}} .
\end{aligned}
$$

One can immediately note that the second-order correction to the current, $\mathbf{j}^{(2)}$, does not play any role.
In addition, since the vector potential is already a secondorder quantity, the terms that contain A in Eqs. (24) and (25) are actually of higher order and can therefore be neglected. We thus define the quantities

$$
\begin{aligned}
\tilde{\mathbf{j}}^{(0)} & =\frac{i \hbar}{2 m}\left(\phi \nabla \phi^{\dagger}-\phi^{\dagger} \nabla \phi\right)+\frac{\hbar}{2 m} \nabla \wedge\left(\phi^{\dagger} \boldsymbol{\sigma} \phi\right) \\
& \equiv \tilde{\mathbf{j}}_{\text {orb }}^{(0)}+\tilde{\mathbf{j}}_{\text {spin }}^{(0)},
\end{aligned}
$$

and

$$
\begin{aligned}
\tilde{\rho}^{(2)}= & \frac{\hbar^{2}}{8 m^{2} c^{2}} \nabla \cdot \nabla\left(\phi^{\dagger} \phi\right) \\
& \left.-\frac{i \hbar^{2}}{8 m^{2} c^{2}} \nabla \cdot\left(\phi^{\dagger} \sigma \wedge(\nabla \phi)+\left(\nabla \phi^{\dagger}\right) \wedge \sigma \phi\right)\right) \\
\equiv & \tilde{\rho}_{\text {orb }}^{(2)}+\tilde{\rho}_{\text {spin }}^{(2)}
\end{aligned}
$$

where the subscripts "orb" and "spin" denote, respectively, the orbital and the spin part of the sources. The modified sources are associated to the mean-field potentials $\tilde{\Phi}^{(2)}$ and $\tilde{\mathbf{A}}^{(2)}$ expressed as

$$
\begin{gathered}
\Phi^{(0)}=\frac{q}{4 \pi \epsilon_{0}} \sum_{i=1}^{N} \int \frac{d \mathbf{x}^{\prime} \rho_{i}^{(0)}}{\left|\mathbf{x}-\mathbf{x}^{\prime}\right|} \\
\tilde{\Phi}^{(2)}=\frac{q}{4 \pi \epsilon_{0}} \sum_{i=1}^{N} \int \frac{d \mathbf{x}^{\prime} \tilde{\rho}_{i}^{(2)}}{\left|\mathbf{x}-\mathbf{x}^{\prime}\right|} \\
\tilde{\mathbf{A}}^{(2)}=\frac{q}{4 \pi \epsilon_{0} c^{2}} \sum_{i=1}^{N} \int d \mathbf{x}^{\prime}\left(\frac{\tilde{\mathbf{j}}(0)}{2\left|\mathbf{x}-\mathbf{x}^{\prime}\right|}+\frac{\mathbf{r}\left(\mathbf{r} \cdot \tilde{\mathbf{j}}_{i}^{(0)}\right)}{2\left|\mathbf{x}-\mathbf{x}^{\prime}\right|^{3}}\right)
\end{gathered}
$$

Finally, a self-consistent mean-field theory at second order in powers of $1 / c$ is given by the following equations:

$$
\begin{gathered}
-\Delta \Phi^{(0)}=\frac{q \rho^{(0)}}{\epsilon_{0}}, \\
-\Delta \tilde{\mathbf{A}}^{(2)}=\frac{q \tilde{\mathbf{j}}_{T}^{(0)}}{\epsilon_{0} c^{2}}, \\
-\Delta \tilde{\Phi}^{(2)}=\frac{q \tilde{\rho}^{(2)}}{\epsilon_{0}}, \\
i \hbar \frac{\partial \phi}{\partial t}=\left(\frac{\mathbf{p}^{2}}{2 m}+m c^{2}+U_{\mathrm{eff}}^{s c}\right) \phi,
\end{gathered}
$$

where

$$
\begin{aligned}
U_{\mathrm{eff}}^{s c}= & q \Phi^{(0)}+q \tilde{\Phi}^{(2)}-\frac{q}{m} \tilde{\mathbf{A}}^{(2)} \cdot \mathbf{p}-\frac{q \hbar}{2 m} \boldsymbol{\sigma} \cdot \nabla \wedge \tilde{\mathbf{A}}^{(2)} \\
& +\frac{q \hbar^{2}}{8 m^{2} c^{2}} \Delta \Phi^{(0)}+\frac{q \hbar}{4 m^{2} c^{2}} \boldsymbol{\sigma} \cdot \nabla \Phi^{(0)} \wedge \mathbf{p}
\end{aligned}
$$

The transformation of Eq. (22) into Eq. (40) is performed using the identities (A1)-(A3) given in Appendix A.

The next step is to obtain the integral form of Eq. (40) by plugging the expressions for the sources [Eqs. (23), (31), and (32)] into the equations of the potentials, given by Eqs. (36)(38). This can be done as follows. 
(1) For the three terms involving $\Phi^{(0)}(\mathbf{x})$ in Eq. (40), we just need to insert Eq. (23) into Eq. (36), leading to

$$
\begin{gathered}
q \Phi^{(0)}=\bar{e}^{2} \sum_{i=1}^{N} \int \frac{d \mathbf{x}^{\prime} \phi_{i}^{\dagger} \phi_{i}}{\left|\mathbf{x}-\mathbf{x}^{\prime}\right|} \\
\frac{q \hbar^{2}}{8 m^{2} c^{2}} \Delta \Phi^{(0)}=\frac{\bar{e}^{2} \hbar^{2}}{8 m^{2} c^{2}} \Delta\left(\sum_{i=1}^{N} \int \frac{d \mathbf{x}^{\prime} \phi_{i}^{\dagger} \phi_{i}}{\left|\mathbf{x}-\mathbf{x}^{\prime}\right|}\right), \\
\frac{q \hbar \boldsymbol{\sigma}}{4 m^{2} c^{2}} \cdot \nabla \Phi^{(0)} \wedge \mathbf{p}=\frac{\bar{e}^{2} \hbar \boldsymbol{\sigma}}{4 m^{2} c^{2}} \cdot \nabla\left(\sum_{i=1}^{N} \int \frac{d \mathbf{x}^{\prime} \phi_{i}^{\dagger} \phi_{i}}{\left|\mathbf{x}-\mathbf{x}^{\prime}\right|}\right) \wedge \mathbf{p} .
\end{gathered}
$$

Equation (41) is the usual Hartree mean-field energy. The righthand side of Eq. (42), originating from the Darwin interaction, is a kind of mean "contact term," while Eq. (43) represents the spin-orbit interaction with the mean electric field created by all the electrons.

(2) In order to determine $\tilde{\Phi}^{(2)}(\mathbf{x})$, we must plug Eq. (32) into Eq. (34). In addition, in order that the space operators act on $\frac{1}{\left|\mathbf{x}-\mathbf{x}^{\prime}\right|}$, the expressions of the two terms of $\tilde{\rho}^{(2)}$ are transformed using the identities (A4) and (A5) given in Appendix A. This leads to the following expression of $q \tilde{\Phi}^{(2)}(\mathbf{x})$ :

$$
\begin{aligned}
q \tilde{\Phi}^{(2)}= & \frac{\bar{e}^{2} \hbar^{2}}{8 m^{2} c^{2}} \sum_{i=1}^{N} \int d \mathbf{x}^{\prime} \phi_{i}^{\dagger} \phi_{i} \Delta \frac{1}{\left|\mathbf{x}-\mathbf{x}^{\prime}\right|}-\frac{\bar{e}^{2} \hbar}{4 m^{2} c^{2}} \\
& \times \sum_{i=1}^{N} \int d \mathbf{x}^{\prime} \phi_{i}^{\dagger} \boldsymbol{\sigma}_{i} \cdot\left(\nabla \frac{1}{\left|\mathbf{x}-\mathbf{x}^{\prime}\right|} \wedge \mathbf{p} \phi_{i}\right) .
\end{aligned}
$$

The first term in the above expression is also a kind of mean contact term. The second term represents a mean "spin-orbit" interaction for which the electric field is the mean field created by all the electrons of the system.

(3) Finally, the last two terms involving the vector potential $\tilde{\mathbf{A}}^{(2)}$, i.e., the paramagnetic term $-\frac{q}{m} \tilde{\mathbf{A}}^{(2)}(\mathbf{x}) \cdot \mathbf{p}$ and the Zeeman interaction $-\frac{q \hbar}{2 m} \boldsymbol{\sigma} \cdot \nabla \wedge \tilde{\mathbf{A}}^{(2)}(\mathbf{x})$, are obtained by plugging Eq. (31) into Eq. (35). Each of them will generate four contributions. Indeed, $\tilde{\mathbf{A}}^{(2)}$ is made of two terms $\frac{\tilde{\mathbf{j}}^{(0)}}{2\left|\mathbf{x}-\mathbf{x}^{\prime}\right|}$ and $\frac{\mathbf{r}\left(\mathbf{r} \tilde{\mathbf{j}}^{(0)}\right)}{2\left|\mathbf{x}-\mathbf{x}^{\prime}\right|^{3}}$, which are themselves composed of the orbital current $\tilde{\mathbf{j}}_{\text {orb }}^{(0)}=\frac{i \hbar}{2 m}\left(\phi \nabla \phi^{\dagger}-\phi^{\dagger} \nabla \phi\right)$ and the spin current $\tilde{\mathbf{j}}_{\text {spin }}^{(0)}=\frac{\hbar}{2 m} \nabla \wedge$ $\left(\phi^{\dagger} \sigma \phi\right)$. Their analytical expressions have been obtained by using the identities (A6) and (A7) given in Appendix A, and may be expressed as

$$
\begin{aligned}
-\frac{q}{m} \tilde{\mathbf{A}}^{(2)} \cdot \mathbf{p} & =-\frac{\bar{e}^{2}}{4 m^{2} c^{2}} \sum_{i=1}^{N}\left[\int d \mathbf{x}^{\prime}\left(\frac{2 \phi_{i}^{\dagger} \mathbf{p} \phi_{i}}{\left|\mathbf{x}-\mathbf{x}^{\prime}\right|}+\frac{2 \mathbf{r}\left(\phi_{i}^{\dagger} \mathbf{p} \phi_{i} \cdot \mathbf{r}\right)}{\left|\mathbf{x}-\mathbf{x}^{\prime}\right|^{3}}+\frac{\hbar \nabla \wedge\left(\phi_{i}^{\dagger} \boldsymbol{\sigma}_{i} \phi_{i}\right)}{\left|\mathbf{x}-\mathbf{x}^{\prime}\right|}+\frac{\hbar \mathbf{r}\left(\nabla \wedge\left(\phi_{i}^{\dagger} \boldsymbol{\sigma}_{i} \phi_{i}\right) \cdot \mathbf{r}\right)}{\left|\mathbf{x}-\mathbf{x}^{\prime}\right|^{3}}\right)\right] \cdot \mathbf{p}, \quad(45) \\
-\frac{q \hbar}{2 m} \boldsymbol{\sigma} \cdot \nabla \wedge \tilde{\mathbf{A}}^{(2)} & =-\frac{\bar{e}^{2} \hbar}{8 m^{2} c^{2}} \sigma \cdot \nabla \wedge \sum_{i=1}^{N}\left[\int d \mathbf{x}^{\prime}\left(\frac{2 \phi_{i}^{\dagger} \mathbf{p} \phi_{i}}{\left|\mathbf{x}-\mathbf{x}^{\prime}\right|}+\frac{2 \mathbf{r}\left(\phi_{i}^{\dagger} \mathbf{p} \phi_{i} \cdot \mathbf{r}\right)}{\left|\mathbf{x}-\mathbf{x}^{\prime}\right|^{3}}+\frac{\hbar \nabla \wedge\left(\phi_{i}^{\dagger} \boldsymbol{\sigma}_{i} \phi_{i}\right)}{\left|\mathbf{x}-\mathbf{x}^{\prime}\right|}+\frac{\hbar \mathbf{r}\left(\nabla \wedge\left(\phi_{i}^{\dagger} \boldsymbol{\sigma}_{i} \phi_{i}\right) \cdot \mathbf{r}\right)}{\left|\mathbf{x}-\mathbf{x}^{\prime}\right|^{3}}\right)\right] .
\end{aligned}
$$

In the paramagnetic term of Eq. (45), the contribution of the orbital current leads to mean "spin-free" terms involving the coupling between the electron momenta, whereas the spin part of the current produces mean "spin-other-orbit" terms. For the Zeeman interaction operator [Eq. (46)], the orbital current contributes to another mean "spin-other-orbit" term and the spin current generates mean "spin-spin" interactions.

Summing up Eqs. (41)-(46), we get the integral form of $U_{\mathrm{eff}}^{s c}(\mathbf{x})$, which represents the mean interaction at second order in $1 / c$, with the following physical interpretations for each term:

$$
\begin{aligned}
& U_{\text {eff }}^{s c}(\mathbf{x})=\bar{e}^{2} \underbrace{\sum_{i=1}^{N} \int \frac{d \mathbf{x}^{\prime} \phi_{i}^{\dagger}\left(\mathbf{x}^{\prime}\right) \phi_{i}\left(\mathbf{x}^{\prime}\right)}{\left|\mathbf{x}-\mathbf{x}^{\prime}\right|}}_{\text {Hartree [Eq. (41)] }}+\frac{\bar{e}^{2} \hbar^{2}}{8 m^{2} c^{2}} \underbrace{\Delta\left(\sum_{i=1}^{N} \int \frac{d \mathbf{x}^{\prime} \phi_{i}^{\dagger}\left(\mathbf{x}^{\prime}\right) \phi_{i}\left(\mathbf{x}^{\prime}\right)}{\left|\mathbf{x}-\mathbf{x}^{\prime}\right|}\right)}_{\text {Contact [Eq. (42)] }}+\frac{\bar{e}^{2} \hbar^{2}}{8 m^{2} c^{2}} \underbrace{\sum_{i=1}^{N} \int d \mathbf{x}^{\prime} \phi_{i}^{\dagger}\left(\mathbf{x}^{\prime}\right) \phi_{i}\left(\mathbf{x}^{\prime}\right) \Delta \frac{1}{\left|\mathbf{x}-\mathbf{x}^{\prime}\right|}}_{\text {Contact [Eq. (44)] }} \\
& +\frac{\bar{e}^{2} \hbar}{4 m^{2} c^{2}} \underbrace{\boldsymbol{\sigma} \cdot \nabla\left(\sum_{i=1}^{N} \int \frac{d \mathbf{x}^{\prime} \phi_{i}^{\dagger}\left(\mathbf{x}^{\prime}\right) \phi_{i}\left(\mathbf{x}^{\prime}\right)}{\left|\mathbf{x}-\mathbf{x}^{\prime}\right|}\right) \wedge \mathbf{p}}_{\text {Spin orbit [Eq. (43)] }}+\frac{\bar{e}^{2} \hbar}{4 m^{2} c^{2}} \underbrace{\sum_{i=1}^{N} \int d \mathbf{x}^{\prime} \phi_{i}^{\dagger}\left(\mathbf{x}^{\prime}\right) \boldsymbol{\sigma}_{i} \cdot\left(\boldsymbol{\nabla} \frac{1}{\left|\mathbf{x}-\mathbf{x}^{\prime}\right|} \wedge \mathbf{p} \phi_{i}\left(\mathbf{x}^{\prime}\right)\right)}_{\text {Spin orbit [Eq. (44)] }} \\
& -\frac{\bar{e}^{2}}{2 m^{2} c^{2}} \underbrace{\sum_{i=1}^{N}\left[\int d \mathbf{x}^{\prime}\left(\frac{\phi_{i}^{\dagger}\left(\mathbf{x}^{\prime}\right) \mathbf{p} \phi_{i}\left(\mathbf{x}^{\prime}\right)}{\left|\mathbf{x}-\mathbf{x}^{\prime}\right|}\right)+\int d \mathbf{x}^{\prime}\left(\frac{\mathbf{r}\left(\phi_{i}^{\dagger}\left(\mathbf{x}^{\prime}\right) \mathbf{p} \phi_{i}\left(\mathbf{x}^{\prime}\right) \cdot \mathbf{r}\right)}{\left|\mathbf{x}-\mathbf{x}^{\prime}\right|^{3}}\right)\right] \cdot \mathbf{p}}_{\text {Spin free [Eq. (45)] }} \\
& -\frac{\hbar \bar{e}^{2}}{4 m^{2} c^{2}} \underbrace{\sum_{i=1}^{N}\left[\int d \mathbf{x}^{\prime}\left(\frac{\nabla \wedge\left[\phi_{i}^{\dagger}\left(\mathbf{x}^{\prime}\right) \boldsymbol{\sigma}_{i} \phi_{i}\left(\mathbf{x}^{\prime}\right)\right]}{\left|\mathbf{x}-\mathbf{x}^{\prime}\right|}\right)+\int d \mathbf{x}^{\prime}\left(\frac{\mathbf{r}\left(\nabla \wedge\left[\phi_{i}^{\dagger}\left(\mathbf{x}^{\prime}\right) \boldsymbol{\sigma}_{i} \phi_{i}\left(\mathbf{x}^{\prime}\right)\right] \cdot \mathbf{r}\right)}{\left|\mathbf{x}-\mathbf{x}^{\prime}\right|^{3}}\right)\right] \cdot \mathbf{p}}
\end{aligned}
$$




$$
\begin{aligned}
& -\frac{\bar{e}^{2} \hbar}{4 m^{2} c^{2}} \underbrace{\boldsymbol{\sigma} \cdot \nabla \wedge \sum_{i=1}^{N}\left[\int d \mathbf{x}^{\prime}\left(\frac{\phi_{i}^{\dagger}\left(\mathbf{x}^{\prime}\right) \mathbf{p} \phi_{i}\left(\mathbf{x}^{\prime}\right)}{\left|\mathbf{x}-\mathbf{x}^{\prime}\right|}\right)+\int d \mathbf{x}^{\prime}\left(\frac{\mathbf{r}\left(\phi_{i}^{\dagger}\left(\mathbf{x}^{\prime}\right) \mathbf{p} \phi_{i}\left(\mathbf{x}^{\prime}\right) \cdot \mathbf{r}\right)}{\left|\mathbf{x}-\mathbf{x}^{\prime}\right|^{3}}\right)\right]}_{\text {Spin-other-orbit [Eq. (46)] }} \\
& -\frac{\bar{e}^{2} \hbar^{2}}{8 m^{2} c^{2}} \underbrace{\boldsymbol{\sigma} \cdot \nabla \wedge \sum_{i=1}^{N}\left[\int d \mathbf{x}^{\prime}\left(\frac{\nabla \wedge\left[\phi_{i}^{\dagger}\left(\mathbf{x}^{\prime}\right) \boldsymbol{\sigma}_{i} \phi_{i}\left(\mathbf{x}^{\prime}\right)\right]}{\left|\mathbf{x}-\mathbf{x}^{\prime}\right|}+\frac{\mathbf{r}\left(\nabla \wedge\left[\phi_{i}^{\dagger}\left(\mathbf{x}^{\prime}\right) \boldsymbol{\sigma}_{i} \phi_{i}\left(\mathbf{x}^{\prime}\right)\right] \cdot \mathbf{r}\right)}{\left|\mathbf{x}-\mathbf{x}^{\prime}\right|^{3}}\right)\right]}_{\text {Spin-spin [Eq. (46)] }} .
\end{aligned}
$$

The physical interpretation of each term and the relevant equation number are given under the horizontal braces; for instance, "Hartree [Eq. (41)]" means that this is the Hartree term derived in Eq. (41). A summary of the nature and origin of each interaction term is provided in Table I.

Now, the crucial point is that each term in Eq. (6) can be shown to be identical to the corresponding term in the Hartree-Breit-Pauli Hamiltonian of Eq. (6). The mathematical procedure to show this equivalence is tedious but straightforward and can be found in Appendix B. Therefore, we have proven that the semirelativistic limit of the self-consistent Dirac-Maxwell equations is completely equivalent to the more standard Hartree-Breit-Pauli mean-field approach.

An important advantage of the above procedure is that one can point out the precise origin of the different kinds of interaction terms (spin free, spin orbit, and spin-spin). Indeed, each term comes from a light-matter interaction operator (Coulomb, Darwin, etc.) and a source term $\left(\rho^{(0)}, \tilde{\mathbf{j}}_{\text {orb }}^{(0)}\right.$, etc.), as is illustrated in Table I. For instance, we can identify that the spin-other-orbit terms are related to two different mechanisms: the paramagnetic coupling between the electron momentum and the spin current, but also the Zeeman interaction involving the orbital current. Instead, the spin-spin interaction originates from the Zeeman effect where the electromagnetic field is produced by the spin current. Finally, another important result is the role played by the second-order correction of the density, which leads to a contact term and a spin-orbit term. The Hartree-Breit-Pauli equations could not be recovered without this contribution.

\section{CONCLUSIONS}

In this work, we demonstrated the equivalence between the Hartree-Breit-Pauli model and the nonrelativistic limit (up to second order in $1 / c$ ) of the self-consistent Dirac-Maxwell equations. We also explained how the charge and current densities that act as sources in the Maxwell equations are related to the microscopic two-electron interactions of the Breit-Pauli model (spin orbit, spin-other-orbit, and spin-spin) via the one-electron light-matter operators appearing in the mean-field Hamiltonian. In particular, the spin current and the second-order correction to the charge density [9] are crucial for obtaining all the spin-dependent terms.

The self-consistent Dirac-Maxwell approach may prove useful to study the ultrafast electron and spin dynamics in nanometric devices (nanoparticles, thin films, quantum wells, etc.) excited by ultrashort and intense laser pulses in the femtoor attosecond domain, particularly for the interpretation of time-resolved magneto-optical experiments. Other possible areas of applications involve dense plasmas and astrophysical systems under extreme conditions of density and pressure [3].

\section{ACKNOWLEDGMENTS}

The authors would like to thank Dr. J.-Y. Bigot and Professor M. Mamaache for helpful comments and discussions. This work was partially funded by the European Research Council (ERC grant “ATOMAG” ERC-2009-AdG-20090325 \#247452) and by the Agence Nationale de la Recherche, project Labex "Nanostructures in Interaction with their Environment."

\section{APPENDIX A: MATHEMATICAL COMPLEMENTS}

In this Appendix we present some mathematical details: (i) the transformation of Eq. (22) into Eq. (40), (ii) the derivation of Eq. (44), and (iii) the derivation of Eqs. (45) and (46). In the following, for the sake of simplicity, the summation symbol $\sum$ has been neglected.

\begin{tabular}{|c|c|c|c|c|c|}
\hline \multirow[b]{2}{*}{ Sources } & \multicolumn{5}{|c|}{ Operators } \\
\hline & $\begin{array}{l}\text { Coulomb } \\
\qquad \Phi\end{array}$ & $\begin{array}{l}\text { Darwin } \\
\frac{q \hbar^{2}}{8 m^{2} c^{2}} \Delta \Phi\end{array}$ & $\begin{array}{c}\text { Spin orbit } \\
\frac{q \hbar}{4 m^{2} c^{2}} \boldsymbol{\sigma} \cdot \nabla \Phi \wedge \mathbf{p}\end{array}$ & $\begin{array}{l}\text { Paramagnetic } \\
\qquad-\frac{q}{m} \mathbf{A} \cdot \mathbf{p}\end{array}$ & $\begin{array}{c}\text { Zeeman } \\
-\frac{q \hbar}{2 m} \boldsymbol{\sigma} \cdot(\boldsymbol{\nabla} \wedge \mathbf{A})\end{array}$ \\
\hline$\rho^{(0)}$ & Hartree [Eq. (41)] & Contact [Eq. (42)] & Spin orbit [Eq. (43)] & & \\
\hline$\tilde{\rho}_{\mathrm{orb}}^{(2)}$ & Contact [Eq. (44)] & & & & \\
\hline$\tilde{\rho}_{\text {spin }}^{(2)}$ & Spin orbit [Eq. (44)] & & & & \\
\hline$\tilde{\mathbf{j}}_{\text {orb }}^{(0)}$ & & & & Spin free [Eq. (45)] & Spin-other-orbit [Eq. (46)] \\
\hline$\tilde{\mathbf{j}}_{\text {spin }}^{(0)}$ & & & & Spin-other-orbit [Eq. (45)] & Spin-spin [Eq. (46)] \\
\hline
\end{tabular}

TABLE I. Origin of the different types of interaction terms in the semirelativistic mean-field Dirac-Maxwell equations. 
(i) The transformation of Eq. (22) into Eq. (40) is performed by using the following three identities:

$$
\begin{gathered}
\nabla \cdot \nabla \Phi^{(0)}=\Delta \Phi^{(0)}, \\
\left(\tilde{\mathbf{A}}^{(2)} \cdot \mathbf{p}+\mathbf{p} \cdot \tilde{\mathbf{A}}^{(2)}\right) \phi=2 \tilde{\mathbf{A}}^{(2)} \cdot \mathbf{p} \phi-i \hbar\left(\nabla \cdot \tilde{\mathbf{A}}^{(2)}\right) \phi=2 \tilde{\mathbf{A}}^{(2)} \cdot \mathbf{p} \phi, \\
\left(\nabla \Phi^{(0)} \wedge \mathbf{p}-\mathbf{p} \wedge \nabla \Phi^{(0)}\right) \phi=2 \nabla \Phi^{(0)} \wedge \mathbf{p} \phi-i \hbar \nabla \wedge\left(\nabla \Phi^{(0)}\right) \phi=2 \nabla \Phi^{(0)} \wedge \mathbf{p} \phi .
\end{gathered}
$$

The identity (A1) is obvious and concerns the Darwin interaction $-\frac{q \hbar^{2}}{8 m^{2} c^{2}} \boldsymbol{\nabla} \cdot \mathbf{E}$ with $\mathbf{E} \equiv-\nabla \Phi^{(0)}$. Then, the paramagnetic term $(\mathbf{p} \cdot \mathbf{A}+\mathbf{A} \cdot \mathbf{p})$ originating from the kinetic-energy operator $\frac{(\mathbf{p}-q \mathbf{A})^{2}}{2 m}$ is transformed thanks to the identity (A2) (where $\left.\mathbf{A} \equiv \tilde{\mathbf{A}}^{(2)}\right)$ and the property of the Coulomb gauge $\nabla \cdot \mathbf{A}=0$. The operator $\frac{\mathbf{A}^{2}}{2 m}$ was neglected because it is of higher order in $1 / c$. Finally, the identity (A3) is needed to transform the spin-orbit interaction $-\frac{q \hbar}{8 m^{2} c^{2}} \boldsymbol{\sigma} \cdot(\mathbf{E} \wedge(\mathbf{p}-q \mathbf{A})-(\mathbf{p}-q \mathbf{A}) \wedge \mathbf{E})$ with $\nabla \Phi^{(0)} \equiv-\mathbf{E}$ and $\mathbf{p} \rightarrow \mathbf{p}-q \mathbf{A}$.

(ii) Let us now focus on the derivation of Eq. (44) where $q \tilde{\Phi}^{(2)}$ is computed by plugging the second-order density $\tilde{\rho}^{(2)}$ of Eq. (32) into Eq. (34). The main idea is to transform the vector expressions under the integral sign in Eq. (34) so that the derivative operators act on $\frac{1}{\left|\mathbf{x}-\mathbf{x}^{\prime}\right|}$ and not on the wave function. This step is performed by using the following identities (see also Ref. [9]):

$$
\begin{gathered}
\int \frac{d \mathbf{x}^{\prime} \tilde{\rho}_{\text {orb }}^{(2)}}{\left|\mathbf{x}-\mathbf{x}^{\prime}\right|}=\frac{\hbar^{2}}{8 m^{2} c^{2}} \int d \mathbf{x}^{\prime}\left\{\nabla \cdot\left[\nabla\left(\phi^{\dagger} \phi\right)\right]\right\} \frac{1}{\left|\mathbf{x}-\mathbf{x}^{\prime}\right|} \\
=\frac{\hbar^{2}}{8 m^{2} c^{2}} \int d \mathbf{x}^{\prime} \phi^{\dagger} \phi \nabla \cdot \nabla\left(\frac{1}{\left|\mathbf{x}-\mathbf{x}^{\prime}\right|}\right) \\
\int \frac{d \mathbf{x}^{\prime} \tilde{\rho}_{\text {spin }}^{(2)}}{\left|\mathbf{x}-\mathbf{x}^{\prime}\right|}=-\frac{i \hbar^{2}}{8 m^{2} c^{2}} \int d \mathbf{x}^{\prime}\left[\nabla \cdot\left(\phi^{\dagger} \boldsymbol{\sigma} \wedge \nabla \phi+\left(\nabla \phi^{\dagger}\right) \wedge \boldsymbol{\sigma} \phi\right)\right] \frac{1}{\left|\mathbf{x}-\mathbf{x}^{\prime}\right|} \\
=-\frac{i \hbar^{2}}{8 m^{2} c^{2}} \int d \mathbf{x}^{\prime} \phi^{\dagger} \boldsymbol{\sigma} \cdot\left(\nabla \frac{1}{\left|\mathbf{x}-\mathbf{x}^{\prime}\right|} \wedge \nabla-\nabla \wedge \nabla \frac{1}{\left|\mathbf{x}-\mathbf{x}^{\prime}\right|}\right) \phi \\
=-\frac{i \hbar^{2}}{4 m^{2} c^{2}} \int d \mathbf{x}^{\prime} \phi^{\dagger} \boldsymbol{\sigma} \cdot\left(\nabla \frac{1}{\left|\mathbf{x}-\mathbf{x}^{\prime}\right|} \wedge \nabla \phi\right) .
\end{gathered}
$$

The identity (A3) was used to determine the last part of Eq. (A5).

(iii) Finally, Eqs. (45) and (46) are obtained by transforming the orbital current with the help of the following identities:

$$
\begin{gathered}
i \hbar \int d \mathbf{x}^{\prime}\left(\frac{\phi\left[\nabla \phi^{\dagger}\right]}{\left|\mathbf{x}-\mathbf{x}^{\prime}\right|}-\frac{\phi^{\dagger} \nabla \phi}{\left|\mathbf{x}-\mathbf{x}^{\prime}\right|}\right)=2 \int \frac{d \mathbf{x}^{\prime} \phi^{\dagger} \mathbf{p} \phi}{\left|\mathbf{x}-\mathbf{x}^{\prime}\right|}, \\
i \hbar \int d \mathbf{x}^{\prime}\left(\frac{\mathbf{r}\left(\phi \nabla \phi^{\dagger} \cdot \mathbf{r}\right)}{\left|\mathbf{x}-\mathbf{x}^{\prime}\right|^{3}}-\frac{\mathbf{r}\left(\phi^{\dagger} \nabla \phi \cdot \mathbf{r}\right)}{\left|\mathbf{x}-\mathbf{x}^{\prime}\right|^{3}}\right)=2 \int d \mathbf{x}^{\prime} \frac{\mathbf{r}\left(\phi^{\dagger} \mathbf{p} \phi \cdot \mathbf{r}\right)}{\left|\mathbf{x}-\mathbf{x}^{\prime}\right|^{3}} .
\end{gathered}
$$

Indeed, writing $\mathbf{p}=-i \hbar \nabla$ and $\mathbf{p}^{\dagger}=i \hbar \nabla$, the left-hand side of Eq. (A6) can be written as

$$
\begin{aligned}
i \hbar & \int d \mathbf{x}^{\prime}\left(\frac{\phi\left[\nabla \phi^{\dagger}\right]}{\left|\mathbf{x}-\mathbf{x}^{\prime}\right|}-\frac{\phi^{\dagger} \nabla \phi}{\left|\mathbf{x}-\mathbf{x}^{\prime}\right|}\right)=\int d \mathbf{x}^{\prime}\left(\frac{\left[\mathbf{p}^{\dagger} \phi^{\dagger}\right] \phi}{\left|\mathbf{x}-\mathbf{x}^{\prime}\right|}+\frac{\phi^{\dagger} \mathbf{p} \phi}{\left|\mathbf{x}-\mathbf{x}^{\prime}\right|}\right) \\
= & \underbrace{\langle\phi| \frac{\mathbf{p}}{\left|\mathbf{x}-\mathbf{x}^{\prime}\right|}}|\phi\rangle+\langle\phi \underbrace{\left.\left|\frac{\mathbf{p}}{\left|\mathbf{x}-\mathbf{x}^{\prime}\right|}\right| \phi\right\rangle}=2\left\langle\phi\left|\frac{\mathbf{p}}{\left|\mathbf{x}-\mathbf{x}^{\prime}\right|}\right| \phi\right\rangle=2 \int \frac{d \mathbf{x}^{\prime} \phi^{\dagger} \mathbf{p} \phi}{\left|\mathbf{x}-\mathbf{x}^{\prime}\right|} .
\end{aligned}
$$

The same procedure is used to obtain Eq. (A7).

\section{APPENDIX B: TRANSFORMATION OF EQ. (47) INTO EQ. (6)}

In order to prove the equivalence between Eqs. (47) and (6), we recall here some mathematical rules. The derivation operator $\nabla$ that is outside the integral sign acts on $\mathbf{x}$ (noted $\nabla_{\mathbf{x}}$ ) whereas the operator situated inside acts on $\mathbf{x}^{\prime}\left(\right.$ noted $\nabla_{\mathbf{x}^{\prime}}$ ).

\section{Total spin-free terms}

In Eq. (47), the total spin-free terms are represented by the operators subscripted "spin free" (interaction between the momenta) but also by the "contact" terms. One clearly sees that no transformations are required for the spin-free terms, since we have 
directly

$$
(47)_{\text {spin free }}=-\frac{\bar{e}^{2}}{2 m^{2} c^{2}}\left[\int d \mathbf{x}^{\prime}\left(\frac{\phi_{i}^{\dagger} \mathbf{p} \phi_{i}}{\left|\mathbf{x}-\mathbf{x}^{\prime}\right|}+\right)+\int d \mathbf{x}^{\prime}\left(\frac{\mathbf{r}\left(\phi_{i}^{\dagger} \mathbf{p} \phi_{i} \cdot \mathbf{r}\right)}{\left|\mathbf{x}-\mathbf{x}^{\prime}\right|^{3}}\right)\right] \cdot \mathbf{p}=(6)_{\text {spin free }}
$$

The two contact terms can be transformed using the property $\Delta(1 / r)=-4 \pi \delta(\mathbf{r})$, which leads to

$$
\begin{aligned}
(47)_{\text {contact }} & =+\frac{\bar{e}^{2} \hbar^{2}}{8 m^{2} c^{2}} \Delta_{\mathbf{x}}\left(\int \frac{d \mathbf{x}^{\prime} \phi_{i}^{\dagger} \phi_{i}}{\left|\mathbf{x}-\mathbf{x}^{\prime}\right|}\right)+\frac{\bar{e}^{2} \hbar^{2}}{8 m^{2} c^{2}} \int d \mathbf{x}^{\prime} \phi_{i}^{\dagger} \phi_{i} \Delta_{\mathbf{x}^{\prime}} \frac{1}{\left|\mathbf{x}-\mathbf{x}^{\prime}\right|} \\
& =+\frac{\bar{e}^{2} \hbar^{2}}{8 m^{2} c^{2}} \int d \mathbf{x}^{\prime} \phi_{i}^{\dagger} \phi_{i}\left[-4 \pi \delta\left(\mathbf{x}-\mathbf{x}^{\prime}\right)\right]+\frac{\bar{e}^{2} \hbar^{2}}{8 m^{2} c^{2}} \int d \mathbf{x}^{\prime} \phi_{i}^{\dagger} \phi_{i}\left[-4 \pi \delta\left(\mathbf{x}-\mathbf{x}^{\prime}\right)\right] \\
& =-\frac{\pi \hbar^{2} \bar{e}^{2}}{m^{2} c^{2}} \int d \mathbf{x}^{\prime} \phi_{i}^{\dagger} \phi_{i} \delta\left(\mathbf{x}-\mathbf{x}^{\prime}\right) \\
& =(6)_{\text {contact }} .
\end{aligned}
$$

The total spin-free terms of Eqs. (47) and (6) are therefore equivalent.

\section{Spin-orbit and spin-other-orbit terms}

(i) We first start with the spin-orbit terms. The equivalence is also easy to prove since we have $\nabla \frac{1}{r}=-\frac{\mathbf{r}}{r^{3}}$ which leads to

$$
\begin{aligned}
(47)_{\text {spin orbit }} & =+\frac{\bar{e}^{2} \hbar}{4 m^{2} c^{2}} \sigma \cdot \nabla_{\mathbf{x}}\left(\int \frac{d \mathbf{x}^{\prime} \phi_{i}^{\dagger} \phi_{i}}{\left|\mathbf{x}-\mathbf{x}^{\prime}\right|}\right) \wedge \mathbf{p}-\frac{\bar{e}^{2} \hbar}{4 m^{2} c^{2}} \int d \mathbf{x}^{\prime} \phi_{i}^{\dagger} \boldsymbol{\sigma}_{i} \cdot\left(\nabla_{\mathbf{x}^{\prime}} \frac{1}{\left|\mathbf{x}-\mathbf{x}^{\prime}\right|} \wedge \mathbf{p} \phi_{i}\right) \\
& =-\frac{\hbar \bar{e}^{2}}{4 m^{2} c^{2}} \boldsymbol{\sigma} \cdot\left(\int d \mathbf{x}^{\prime} \phi_{i}^{\dagger} \frac{\mathbf{x}-\mathbf{x}^{\prime}}{\left|\mathbf{x}-\mathbf{x}^{\prime}\right|^{3}} \phi_{i}\right) \wedge \mathbf{p}+\frac{\hbar \bar{e}^{2}}{4 m^{2} c^{2}} \int d \mathbf{x}^{\prime} \phi_{i}^{\dagger} \boldsymbol{\sigma}_{i} \cdot\left(\frac{\mathbf{x}-\mathbf{x}^{\prime}}{\left|\mathbf{x}-\mathbf{x}^{\prime}\right|^{3}} \wedge \mathbf{p}_{i}\right) \phi_{i} \\
& =(6)_{\text {spin orbit }} .
\end{aligned}
$$

The spin-orbit terms of Eqs. (47) and (6) are therefore equivalent.

(ii) The proof of the equivalence between the spin-other-orbit terms is less trivial. Let us split the four terms of Eq. (47) into two groups, those factored by the $\operatorname{spin}^{\sigma}(47)_{\text {other orbit }}$ and those factored by the momentum ${ }^{\mathbf{p}}(47)_{\text {other orbit }}$ :

$$
\begin{aligned}
& \boldsymbol{\sigma}_{(47)_{\text {other orbit }}}=-\frac{\bar{e}^{2} \hbar}{4 m^{2} c^{2}} \sigma \cdot \nabla_{\mathbf{x}} \wedge\left[\int d \mathbf{x}^{\prime}\left(\frac{\phi_{i}^{\dagger} \mathbf{p} \phi_{i}}{\left|\mathbf{x}-\mathbf{x}^{\prime}\right|}+\frac{\mathbf{r}\left(\phi_{i}^{\dagger} \mathbf{p} \phi_{i} \cdot \mathbf{r}\right)}{\left|\mathbf{x}-\mathbf{x}^{\prime}\right|^{3}}\right)\right], \\
& \mathbf{p}_{(47)_{\text {other orbit }}}=-\frac{\hbar \bar{e}^{2}}{4 m^{2} c^{2}}\left[\int d \mathbf{x}^{\prime}\left(\frac{\nabla_{\mathbf{x}^{\prime}} \wedge\left(\phi_{i}^{\dagger} \boldsymbol{\sigma}_{i} \phi_{i}\right)}{\left|\mathbf{x}-\mathbf{x}^{\prime}\right|}+\frac{\mathbf{r}\left(\nabla_{\mathbf{x}^{\prime}} \wedge\left(\phi_{i}^{\dagger} \boldsymbol{\sigma}_{i} \phi_{i}\right) \cdot \mathbf{r}\right)}{\left|\mathbf{x}-\mathbf{x}^{\prime}\right|^{3}}\right)\right] \cdot \mathbf{p} .
\end{aligned}
$$

Let us first focus on the former. By using the identity $\mathbf{a} \wedge(\mathbf{b} \wedge \mathbf{c})=(\mathbf{a} \cdot \mathbf{b}) \mathbf{c}-(\mathbf{a} \cdot \mathbf{c}) \mathbf{b}$ the second term $\frac{\mathbf{r}\left(\phi_{i}^{\dagger} \mathbf{p} \phi_{i} \cdot \mathbf{r}\right)}{\left|\mathbf{x}-\mathbf{x}^{\prime}\right|^{3}}$ becomes

$$
\begin{aligned}
\sigma_{(47)_{\text {other orbit }}} & =-\frac{\bar{e}^{2} \hbar}{4 m^{2} c^{2}} \sigma \cdot \nabla_{\mathbf{x}} \wedge\left[\int d \mathbf{x}^{\prime}\left(\frac{\phi_{i}^{\dagger} \mathbf{p} \phi_{i}}{\left|\mathbf{x}-\mathbf{x}^{\prime}\right|}+\frac{\mathbf{r}\left(\phi_{i}^{\dagger} \mathbf{p} \phi_{i} \cdot \mathbf{r}\right)}{\left|\mathbf{x}-\mathbf{x}^{\prime}\right|^{3}}\right)\right] \\
& =-\frac{\bar{e}^{2} \hbar}{4 m^{2} c^{2}} \sigma \cdot\left[\int d \mathbf{x}^{\prime} \nabla_{\mathbf{x}} \wedge\left(2 \frac{\phi_{i}^{\dagger} \mathbf{p} \phi_{i}}{\left|\mathbf{x}-\mathbf{x}^{\prime}\right|}+\frac{\mathbf{r} \wedge\left(\mathbf{r} \wedge \phi_{i}^{\dagger} \mathbf{p} \phi_{i}\right)}{\left|\mathbf{x}-\mathbf{x}^{\prime}\right|^{3}}\right)\right] .
\end{aligned}
$$

Then, by applying the derivative rule $\nabla \wedge(f(x) \mathbf{p})=\nabla f(x) \wedge \mathbf{p}+f(x)(\nabla \wedge \mathbf{p})=\nabla f(x) \wedge \mathbf{p}$ to the first term of Eq. (B1) and by using the relation $\nabla \wedge(\mathbf{b} \wedge \mathbf{c})=(\nabla \cdot \mathbf{b}) \mathbf{c}-(\boldsymbol{\nabla} \cdot \mathbf{c}) \mathbf{b}$ to transform the second term of Eq. (B1), one obtains Eq. (B2), which leads directly to

$$
\begin{aligned}
& { }^{\sigma}(47)_{\text {other orbit }}=-\frac{\bar{e}^{2} \hbar}{4 m^{2} c^{2}} \sigma \cdot \int d \mathbf{x}^{\prime}(\nabla \frac{2}{\nabla \mathbf{x}-\mathbf{x}^{\prime} \mid} \wedge \phi_{i}^{\dagger} \mathbf{p} \phi_{i}+\overbrace{\nabla \cdot\left(\mathbf{r} \wedge \phi_{i}^{\dagger} \mathbf{p} \phi_{i}\right)}^{0} \frac{\mathbf{r}}{\left|\mathbf{x}-\mathbf{x}^{\prime}\right|^{3}}-\overbrace{4 \pi \delta(\mathbf{r})\left(\mathbf{r} \wedge \phi_{i}^{\dagger} \mathbf{p} \phi_{i}\right)}^{0}) \\
& =\frac{\bar{e}^{2} \hbar}{2 m^{2} c^{2}} \boldsymbol{\sigma} \cdot \int d \mathbf{x}^{\prime} \frac{\mathbf{x}-\mathbf{x}^{\prime}}{\left|\mathbf{x}-\mathbf{x}^{\prime}\right|^{3}} \wedge \phi_{i}^{\dagger} \mathbf{p} \phi_{i} .
\end{aligned}
$$


A similar procedure is used to transform $\mathbf{p}(47)_{\text {other orbit }}$, leading to

$$
\begin{aligned}
\mathbf{p}_{(47)_{\text {other orbit }}} & =-\frac{\hbar \bar{e}^{2}}{4 m^{2} c^{2}}\left[\int d \mathbf{x}^{\prime}\left(-2 \nabla \frac{1}{\left|\mathbf{x}-\mathbf{x}^{\prime}\right|} \wedge \phi_{i}^{\dagger} \boldsymbol{\sigma}_{i} \phi_{i}\right)\right] \cdot \mathbf{p} \\
& =-\frac{\hbar \bar{e}^{2}}{2 m^{2} c^{2}}\left[\int d \mathbf{x}^{\prime} \frac{\left(\mathbf{x}-\mathbf{x}^{\prime}\right)}{\left|\mathbf{x}-\mathbf{x}^{\prime}\right|^{3}} \wedge \phi_{i}^{\dagger} \boldsymbol{\sigma}_{i} \phi_{i}\right] \cdot \mathbf{p} .
\end{aligned}
$$

Finally, summing Eqs. (B3) and (B4), the spin-other-orbit terms of Eqs. (47) and (6) are proven to be equivalent:

$$
\begin{aligned}
\sigma(47)_{\text {other orbit }}+{ }^{\mathbf{p}}(47)_{\text {other orbit }}= & \frac{\bar{e}^{2} \hbar}{2 m^{2} c^{2}} \sigma \cdot \int d \mathbf{x}^{\prime} \frac{\mathbf{x}-\mathbf{x}^{\prime}}{\left|\mathbf{x}-\mathbf{x}^{\prime}\right|^{3}} \wedge \phi_{i}^{\dagger} \mathbf{p} \phi_{i} \\
& -\frac{\hbar \bar{e}^{2}}{2 m^{2} c^{2}}\left[\int d \mathbf{x}^{\prime} \frac{\left(\mathbf{x}-\mathbf{x}^{\prime}\right)}{\left|\mathbf{x}-\mathbf{x}^{\prime}\right|^{3}} \wedge \phi_{i}^{\dagger} \boldsymbol{\sigma}_{i} \phi_{i}\right] \cdot \mathbf{p} \\
= & (6)_{\text {other orbit }}
\end{aligned}
$$

\section{Spin-spin terms}

Starting from $(47)_{\text {spin-spin }}$ and using again the relation $\mathbf{a} \wedge(\mathbf{b} \wedge \mathbf{c})=(\mathbf{a} \cdot \mathbf{b}) \mathbf{c}-(\mathbf{a} \cdot \mathbf{c}) \mathbf{b}$ to transform the second term $\frac{\mathbf{r}\left(\nabla \wedge\left(\phi_{i}^{\dagger} \sigma_{i} \phi_{i}\right) \cdot \mathbf{r}\right)}{\left|\mathbf{x}-\mathbf{x}^{\prime}\right|^{3}}$, the spin-spin terms are modified into the following:

$$
\begin{aligned}
(47)_{\text {spin-spin }} & =-\frac{\bar{e}^{2} \hbar^{2}}{8 m^{2} c^{2}} \sigma \cdot \nabla_{\mathbf{x}} \wedge \int d \mathbf{x}^{\prime}\left(\frac{\nabla_{\mathbf{x}^{\prime}} \wedge\left(\phi_{i}^{\dagger} \boldsymbol{\sigma}_{i} \phi_{i}\right)}{\left|\mathbf{x}-\mathbf{x}^{\prime}\right|}+\frac{\mathbf{r}\left(\nabla_{\mathbf{x}^{\prime}} \wedge\left(\phi_{i}^{\dagger} \boldsymbol{\sigma}_{i} \phi_{i} \cdot \mathbf{r}\right)\right.}{\left|\mathbf{x}-\mathbf{x}^{\prime}\right|^{3}}\right) \\
& =-\frac{\bar{e}^{2} \hbar^{2}}{8 m^{2} c^{2}} \boldsymbol{\sigma} \cdot \int d \mathbf{x}^{\prime}\left[2 \nabla_{\mathbf{x}} \wedge\left(\frac{\nabla_{\mathbf{x}^{\prime}} \wedge\left(\phi_{i}^{\dagger} \boldsymbol{\sigma}_{i} \phi_{i}\right)}{\left|\mathbf{x}-\mathbf{x}^{\prime}\right|}\right)+\nabla_{\mathbf{x}} \wedge\left(\frac{\mathbf{r} \wedge\left(\mathbf{r} \wedge \nabla_{\mathbf{x}^{\prime}} \wedge\left(\phi_{i}^{\dagger} \boldsymbol{\sigma}_{i} \phi_{i}\right)\right)}{\left|\mathbf{x}-\mathbf{x}^{\prime}\right|^{3}}\right)\right] .
\end{aligned}
$$

Then, applying the mathematical rule $\nabla \wedge(f(x) \mathbf{p})=\nabla f(x) \wedge \mathbf{p}+f(x)(\nabla \wedge \mathbf{p})$ to the first term of the above expression and using the property $\nabla \wedge(\mathbf{r} \wedge \mathbf{A})=2 \mathbf{A}$ allows us to cancel out the two bracketed terms of Eq. (B6). The remaining term of Eq. (B6) is transformed into Eq. (B7) by using the vector identity $\nabla \wedge \nabla \wedge f=\nabla(\nabla \cdot f)-\Delta f$. Finally, the procedure permitting us to obtain Eq. (B8) from Eq. (B7) is detailed in many textbooks [8,17]:

$$
\begin{aligned}
(47)_{\text {spin-spin }}= & -\frac{\bar{e}^{2} \hbar^{2}}{8 m^{2} c^{2}} \sigma \cdot \int d \mathbf{x}^{\prime}\left(2 \nabla \wedge\left(\nabla \wedge\left(\phi_{i}^{\dagger} \boldsymbol{\sigma}_{i} \phi_{i}\right)\right) \frac{1}{\left|\mathbf{x}-\mathbf{x}^{\prime}\right|}+\left[\frac{-2 \mathbf{r}}{\left|\mathbf{x}-\mathbf{x}^{\prime}\right|^{3}} \wedge\left(\nabla \wedge\left(\phi_{i}^{\dagger} \boldsymbol{\sigma}_{i} \phi_{i}\right)\right]_{\equiv-2 A}\right.\right. \\
& \left.+\left[\nabla \wedge\left(\mathbf{r} \wedge \frac{\mathbf{r}}{\left|\mathbf{x}-\mathbf{x}^{\prime}\right|^{3}} \wedge\left(\nabla \wedge\left(\phi_{i}^{\dagger} \boldsymbol{\sigma}_{i} \phi_{i}\right)\right)\right)\right]_{\equiv \nabla \wedge(\mathbf{r} \wedge \mathbf{A})}\right) \\
& =-\frac{\bar{e}^{2} \hbar^{2}}{4 m^{2} c^{2}} \sigma \cdot \int d \mathbf{x}^{\prime}\left(\nabla\left[\nabla \cdot\left(\phi_{i}^{\dagger} \boldsymbol{\sigma}_{i} \phi_{i}\right)\right] \frac{1}{\left|\mathbf{x}-\mathbf{x}^{\prime}\right|}-\left(\phi_{i}^{\dagger} \boldsymbol{\sigma}_{i} \phi_{i}\right) \Delta \frac{1}{\left|\mathbf{x}-\mathbf{x}^{\prime}\right|}\right) \\
& =-\frac{\bar{e}^{2} \hbar^{2}}{4 m^{2} c^{2}} \sigma \cdot \int d \mathbf{x}^{\prime} \phi_{i}^{\dagger}\left(-8 \pi \frac{\sigma_{j}}{3} \delta\left(\mathbf{x}-\mathbf{x}^{\prime}\right)-\frac{\sigma_{j}}{\left|\mathbf{x}-\mathbf{x}^{\prime}\right|^{3}}+3 \frac{\mathbf{r}\left(\sigma_{j} \cdot \mathbf{r}\right)}{\left|\mathbf{x}-\mathbf{x}^{\prime}\right|^{5}}\right) \phi_{i} \\
& =(6)_{\text {spin-spin }} .
\end{aligned}
$$

The spin-spin terms of Eqs. (47) and (6) are therefore equivalent.

[1] W. R. Johnson, Atomic Structure Theory: Lectures on Atomic Physics (Springer-Verlag, Berlin, 2007).

[2] E. Engel, R. M. Dreizler, S. Varga, and B. Fricke, Relativistic Effects in Heavy-Element Chemistry and Physics (Wiley, New York, 2003).

[3] C. P. Ridgers, C. S. Brady, R. Duclous, J. G. Kirk, K. Bennett, T. D. Arber, and A. R. Bell, Phys. Plasmas 20, 056701 (2013).

[4] J.-Y. Bigot et al., Nature Physics 5, 515 (2009).
[5] O. Morandi, J. Zamanian, G. Manfredi, and P.-A. Hervieux, Phys. Rev. E 90, 013103 (2014).

[6] L. D. Landau and E. M. Lifshitz, The Classical Theory of Fields (Butterworth-Heinemann, Oxford, 1979).

[7] J. D. Jackson, Classical Electrodynamics (Wiley, New York, 1975).

[8] H. A. Bethe and E. E. Salpeter, Quantum Mechanics of Oneand Two-Electron Atoms (Plenum, New York, 1977). 
[9] A. Dixit, Y. Hinschberger, J. Zamanian, G. Manfredi, and P.-A. Hervieux, Phys. Rev. A 88, 032117 (2013).

[10] R. M. Dreizler and E. K. U. Gross, Density Functional Theory (Springer-Verlag, Berlin, 1990).

[11] Y. Hinschberger and P.-A. Hervieux, Phys. Rev. B 88, 134413 (2013).

[12] T. Damour and G. Schäfer, Phys. Rev. D 37, 1099 (1988).

[13] J. J. Sakurai, Advanced Quantum Mechanics (Addison-Wesley, Reading, 1967).

[14] P. Strange, Relativistic Quantum Mechanics (Cambridge University Press, Cambridge, 2005).

[15] G. Breit, Phys. Rev. 34, 375 (1929).

[16] V. B. Berestetskii, E. M. Lifshitz, and L. P. Pitaevskii, Quantum Electrodynamics (Butterworth-Heinemann, Oxford, 1999).
[17] K. G. Dyall and K. Faegri, Jr., Introduction to Relativistic Quantum Chemistry (Oxford University Press, New York, 2007).

[18] M. Reiher and A. Wolf, Relativistic Quantum Chemistry (Wiley, New York, 2009).

[19] S. M. Blinder, Am. J. Phys 33, 431 (1965).

[20] Here the electromagnetic field is treated classically.

[21] T. B. Krause, A. Apte, and P. J. Morrison, Physics of Plasmas 14, 102112 (2007).

[22] J. D. Jackson, Am. J. Phys 70, 917 (2002).

[23] L. L. Foldy and S. A. Wouthuysen, Phys. Rev. 78, 29 (1950).

[24] Y. Hinschberger and P.-A. Hervieux, Phys. Lett. A 376, 813 (2012).

[25] G. Manfredi, Eur. J. Phys. 34, 859 (2013). 